\title{
Methodology for Automatic Identification of Emotions in Learning Environments
}

\author{
Yesenia N. González Meneses ${ }^{1}$, Josefina Guerrero García ${ }^{1}$, \\ Carlos Alberto Reyes García ${ }^{2}$, Iván Olmos Pineda ${ }^{1}$, Juan Manuel González Calleros ${ }^{1}$ \\ ${ }^{1}$ BUAP, Facultad de Computación, Puebla, Mexico \\ ${ }^{2}$ INAOE, Ciencias Computacionales, Puebla, Mexico \\ \{yeseniaglez0, joseguga0, ivanoprkl, jumagoca78\}@gmail.com, \\ kargaxxi@inaoep.mx
}

\begin{abstract}
This paper presents a methodological proposal for automatic identification of emotions in educational environments using machine learning algorithms and physiological and behavioral signal acquisition technologies to identify relations between emotions and learning. Four of the main learningcentered emotions are considered [1]: engagement, boredom, confusion and frustration. It is proposed to make a fusion of data from two physiological and behavioral signal acquisition technologies with the objective of achieving the identification of emotions in the most precise manner. Therefore, considering the stages of the proposed methodology, the first of them is presented and the design of the experiment that will be executed for data collection. The development of an appropriate database with elements belonging to a learning environment for the study of emotions is an essential task.
\end{abstract}

Keywords: automatic identification of emotions, learning-centered emotions, learning environment, physiological signals, machine learning.

\section{Introduction}

From a computational approach, the detection and identification of emotions is a relevant problem within the area of affective computation research, since it is the starting point in the study and development of human-machine interaction systems sensitive to the emotions of human beings. In the human-machine interaction area, this is known as classification of emotions, automatic detection or identification of emotions, as it is mentioned in [2] and [3]. This problem is supported by computational models of emotion. In the case of the psychological research that uses computational models, the emphasis will largely be on fidelity with respect to human emotion processes. In AI (Artificial Intelligent) and Robotics related works, evaluation often emphasizes on how the modeling of emotion impacts reasoning processes or leads in some way to improved performances. In Human-Computer Interaction (HCI) work, the key evaluation is whether the model improves human computer interaction such as making it more effective, efficient or pleasant.

To appreciate the transformative role that computational models of emotion can have on research, in [2] they consider three aspects: the impact on emotion research in 
psychology, the impact on artificial intelligence (AI) and finally the impact on work in human-computer interaction. Thus, the complexity of the problems that affective computation deals with lies in the fact it is an inter-disciplinary area that encompasses computer science, psychology, and cognitive science [4]. Particularly, in the computational area the challenge is selecting and testing machine learning algorithms that could be integrated in a complete model of recognition of learning-centered emotions.

In this sense, two approaches have been used to recognize human emotions [5]: The objective, using sensors or capturing images; as in the recognition of facial expressions, voice, heart rate, body language, body's thermal activity, muscular activity and brain waves. And the subjective, such as contextual analysis through direct observation, surveys, or interrogations of the individuals themselves.

The automatic recognition of emotions and the human-machine interaction can be considered, then, from the use of physiological signal sensors that allow the acquisition of data through voice analysis, video images of the face, eyes, head, or body movements of the people [5].

Within the human-machine interfaces that capture physiological signals that can help in the recognition of emotions, as mentioned in [6], the brain wave diadems that send information to an electronic device in the form of an electroencephalogram (EEG) are found.

There are also cardiovascular wristbands, which measure heart rate and provide information in the form of an electrocardiogram (ECG); electrodermal activity sensors that measure the level of conductivity of the skin through sweat on the hands; thermal cameras that allow the measurement of the temperature change of the human body associated with the different emotional states.

There are also devices that measure muscle electrical activity in response to a nerve stimulation of a muscle, in the form of an electromyography (EMG). Regarding devices related to the identification of people's behavior -such as body postures and gestures- there are traditional video cameras, webcams or augmented reality that allow the recording of facial expressions and body movements, as well as eye tracking, important to the recognition of emotions. In this type of devices, there are also voice recorders, another medium used to identify emotions, as mentioned in [7].

The data obtained from different devices must be processed and classified based on a specific objective. In this case, they will be used for the recognition of learningcentered emotions and captured in real educational environments when students are executing a learning activity. Therefore, it is expected to contribute to physiological signal processing technics, from their capture, preprocessing, selection of characteristics and, finally, with the identification of learning-centered emotions.

The objective of identifying emotions during educational activities is to corroborate the relationship between learning-centered emotions and the level of learning obtained by students. This relationship is the base for approaching educational strategies that help to improve the levels of learning and, therefore, the educational level in our country. 


\section{Motivation}

The problem involving automatic recognition of emotions has been an area of investigation highly active in the last years. Regardless of this, a clear solution, which is within reach of the most people, is still far away. Several drawbacks have influenced the construction of an appropriate solution from a computational point of view. On one hand, a factor that affects the performance of emotion recognizers in real contexts is the difficulty to generate databases with spontaneous emotions. Generally, works are made with actuated databases which provide portraits of emotions representing prototypical and intense emotions that facilitate the search of correlations and the subsequent automatic classification. This kind of databases are usually captured in a controlled environment, which decreases problems in the processing of information (noise, for example).

In addition, it can guarantee a balanced number of samples per class. As consequence, there have not been good results when translating the knowledge extracted from these databases to real contexts [8]. In contrast, the data bases with spontaneous records show information with emotional content that does not belong to a single class, but a mixture of them. In other cases, there are samples with a very light emotional charge, close to a neutral emotional state. In addition, databases with spontaneous emotions are usually recorded in noisy environments, such as classrooms, study rooms, entertainment areas, offices, factories or in phone conversations, which leads to the inclusion of noise.

Finally, because of the very nature of the problem, it is not possible to ensure a balanced quantity of examples per class. Another challenge to be solved is the extraction and selection of a set of characteristics that allow recognizing emotions in the data captured spontaneously. Although progress in the area has been important, there is still much to be done in realistic contexts. Therefore, it is necessary to propose and explore other approaches that allow reaching a good performance of the recognition of emotions in real world applications. An evident aspect to consider is the fact that the area of application has an important influence on the accuracy of emotion recognition [7], as well as the degree of intrusion of the tools used.

\subsection{Problem Statement}

Considering the presented problems and the analysis of recent related works, we can identify that in learning environments there is a lack of adequate methodologies to recognize the emotional state of students during learning processes through interaction with a computer. Preferably, that integrates diversified characteristics, obtained even from the fusion of data coming from the use of different technologies of acquisition of physiological and behavioral signals based on a model of emotions that allows to become closer to reality and, overall, to the cognitive learning process, which contributes to the analysis of the emotion-learning relation.

\subsection{Research Objective}

The objective of this work is to recognize emotions in educational environments using machine learning algorithms and technologies of acquisition of physiological and 
behavioral signals to identify emotion-learning relationships. To achieve the above, the following activities are proposed:

- Investigate, analyze and select the model of emotions and technologies for the acquisition of physiological data on which the proposal will be based.

- Analyze and select educational environments to make the physiological and behavioral data capture.

- Investigate, study and select machine learning algorithms for the selection of characteristics for the classification of emotions.

- Design a methodology for the identification of learning-centered emotions.

To validate the work done, tests will be carried out with college students and the recognition accuracy will be evaluated with metrics that allow comparing it with related works in the literature. With this we pretend to corroborate how the proposed methodology allows identifying emotions in educational contexts that can contribute to decision making in emotion-learning relationships.

\section{State of the Art}

The analysis of the state of the art that is being carried out consists of a collection of articles focused specifically on the recognition of emotions in learning activities and the identification of the emotion-learning relationship. These investigations in automatic recognition of emotions represent approximately $20 \%$, and the remaining proportion (approximately 80\%) are works that recognize basic emotions (happiness, sadness, fear, anger, contempt, disgust and surprise). There is a clear difference in the literature found. There are works that, although they make an identification of emotions, give greater relevance to the analysis of the emotion-learning relationship. Others emphasize especially the algorithms for automatic recognition of emotions and the recognition rate reached and, with less importance, analyze the emotion-learning relationship. Considering these two aspects, a review of the state of the art analyzed so far is presented.

\subsection{Works Focused on the Computational Problem of Automatic Recognition of Learning-centered Emotions}

In the literature reviewed, these works represent approximately $63 \%$ of the total of papers reviewed that identify learning-centered emotions. Only the most recent research is mentioned here.

In the work of [10] they use a convolutional neuronal network for the recognition of learning-centered emotions. They run tests using three data-bases: RaFD, database of posed facial expressions containing images of 8 basic emotions and two spontaneous databases created by them, especially with content related to learningcentered emotions. The emotions they recognize are: engagement, excitement, boredom and relaxation.

In [11] they propose a computer-assisted method for special school instructors, where they teach students with mental disorders or emotional problems using a system that employs wearable sensor technologies and intelligent recognition of 
emotions. The emotion recognition module starts with the capture of the signal from brain diadems. The filtered data is sent to the characteristic extraction module. After extracting the characteristics, they are processed with two classifiers: support vector machine and near k-neighbors with a cross-validation of 10 iterations. The emotions they recognize are: happiness, calm, sadness and fear.

In [12] they implement a binary local pattern for the recognition of learningcentered emotions. The purpose of the work is to build a database of spontaneous facial expressions corresponding to affective states in learning environments to be used in different intelligent tutorial systems. The data capture technologies they use are video and EEG diadems (Emotiv-EPOC). The learning-centered emotions that they recognize are: frustration, boredom, engagement and excitement. They use a support vectors machine to do the classification of emotions

The work of [2] explains the construction and validation of a database of facial expressions that they collect by taking pictures with a webcam. Each photograph is labeled with the emotions of the users obtained at that moment from the EmotivEPOC device. For the recognition of facial expressions, they use a technique based on geometry. These measurements are transformed into characteristics to train a support vector machine. They obtain accuracy, per emotion, of: boredom of $64 \%$, engagement of $64 \%$, excitement of $83 \%$ and frustration of $62 \%$. The same model for the recognition of emotions is also used in [13], as part of an affective learning environment based on Web 3.0 to learn how to program in Java.

Finally, in [14] they propose different recommended activities to induce a certain mental state and capture the EEG response for each one of them. They intend to identify the ideal emotional state to learn. These activities are based on psychological research dedicated to measuring the level of attention, concentration and other functions. In the classification process, they use k-means and clustering.

Of the totality of works reviewed we can conclude that the most popular devices for the capture of data are webcams, followed by EEG Emotiv diadems. From the use of these technologies and other less popular ones, $80 \%$ of the works create their own databases to train and assess their recognizers. The most used algorithms for classification tasks are artificial neural networks, support vector machine (SVM), clustering techniques, Bayesian classifiers and nearer neighbors (KNN), among others. Considering this analysis, we will begin the processing of the data collected using the aforementioned algorithms on the signals that we capture.

\subsection{Works Focused on the Analysis of the Emotion-learning Relationship based on the Automatic Recognition of Learning-centered Emotions}

Up next, the most recent research on the analysis of the emotion-learning relationship using computer techniques for the identification of emotions is listed. These works represent $37 \%$ of the literature reviewed so far.

In [15], they make a study of the affective states that originate when students learn with technology, using a tutorial to learn the basics of programming in Python. The emotions they can identify are: engagement, confusion, frustration, boredom, curiosity (which were the most frequent affective states), anxiety, happiness, anguish, surprise, disgust, sadness and fear (which were the rarest). With the analysis of their results, they identify the emotion-learning relationship from five different approaches. 
In [16] they present the development of an intelligent tutor with recognition and management of emotions for mathematics. And in [17] they develop an affective learning system for algorithmic logic by applying gamification. In both works, a module for the recognition of emotions is integrated. This process is based on the analysis of faces.

The analysis of these works helped us to identify the areas of opportunity regarding our research and to be able to define the design strategies of the methodology and algorithms for the selection of characteristics and classification to be used. The above, to validate the hypothesis that states that the proposed methodology allows us to identify emotions in educational contexts that contribute to the decision-making in the emotion-learning relationship.

\section{$4 \quad$ Proposed Methodology}

General methodology of the investigation is shown in Fig.1.

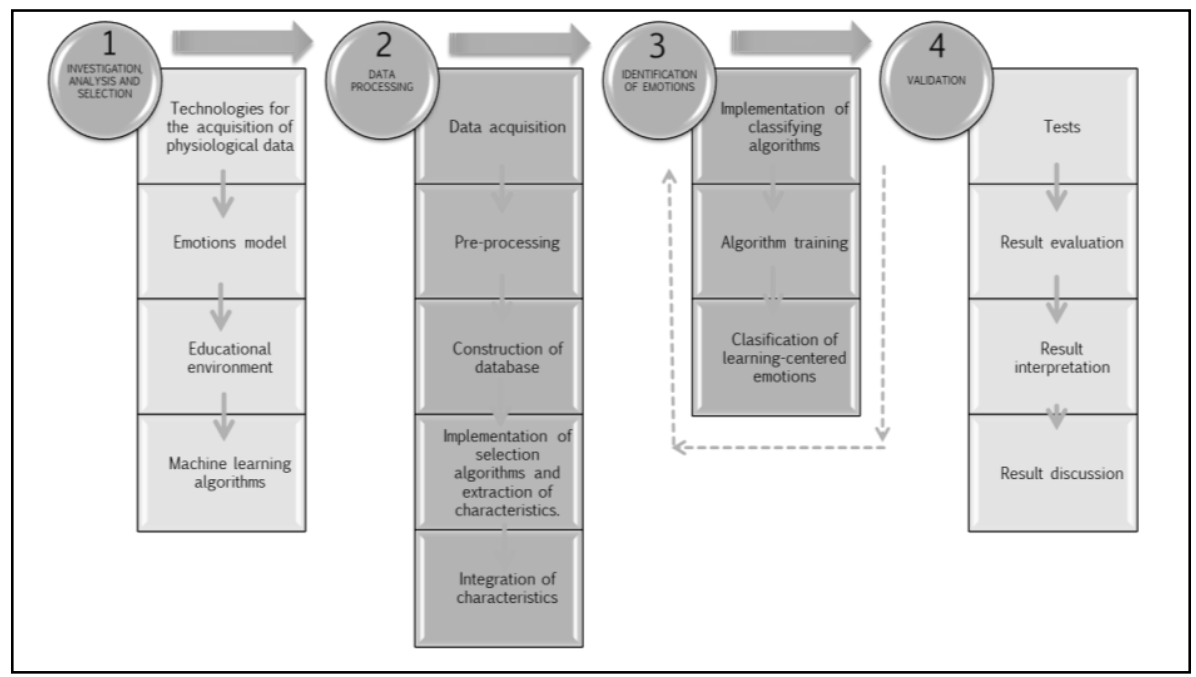

Fig. 1. Proposed Methodology.

The main stages of this methodology are:

1. Research, analysis and selection of technologies for acquisition of physiological data, emotions model, application context and machine learning algorithms, which will be proposed for the development of the methodology.

2. Data processing; it includes the recording and acquisition of physiological and behavioral data to form the data base, the preprocessing required to prepare the data and the implementation of extraction, selection and integration algorithms of relevant characteristics derived from the different signals. We will create a database of student features obtained in real time while doing a learning activity. Which will be formed by two physiological characteristics: the temperature of 
the face and the heart rate; and two behavioral characteristics: the facial image and the movements of the superior limbs.

3. Identification of emotions; the selected machine learning algorithms will be tested and trained for classifying learning-centered emotions. We will start with neural networks adjusted to the characteristic vectors formed by the aforementioned data, trying to recognize emotions from a discrete approach. We will also test fuzzy classification algorithms with the objective of recognizing emotions from a continuous focus. In both cases, with the purpose of improving recognition accuracy with respect to other similar jobs in the state of the art.

4. Validation; tests will be carried out to evaluate the complete methodology for the identification of emotions with metrics that measure their execution, precision and accuracy.

In the stages of data processing and identification of emotions we will follow the steps of the KDD process (Knowledge Discovery in Databases), which correspond to each of the activities considered in the stages of the methodology.

\section{Discussion}

For the development of the proposed methodology, the use of machine learning algorithms is proposed, starting with neural networks and the use of fuzzy techniques. A preliminary analysis of the captured data will allow us to choose the best algorithms according to their characteristics and distribution. A contribution is expected with the proposal of integrating data from different physiological and behavioral signal acquisition technologies.

The data acquisition stage will be carried out through a controlled experiment, in which college students will participate using a tutorial to learn algebra. Data of 100 students will be collected. It is expected to obtain results that contribute to the improvement of the identification of learning-centered emotions with better precision and that can be considered for the decision-making process on pedagogical strategies and learning activities more adapted to each student.

\section{Conclusion}

The development of this project will contribute to the identification of gaps in the automatic recognition of learning-centered emotions. It will allow identifying and proposing less uncomfortable technologies for students, recommended for the collection of data in the identification of learning-centered emotions; we will start with the integration of video cameras, thermal cameras, heart rate sensor and Kinect for Windows.

The implementation of the process of automatic identification of emotions can be integrated into learning environments within the student modeling module or within another type of environment, which may consider the emotional state to provide feedback to the students' learning. 


\section{References}

1. D’Mello, S., Graesser, A.: Dynamics of affective states during complex learning. Learn. Instr. 22(2), pp. 145-157 (2012)

2. Armony, J.: Computational models of emotion. In: Proceedings. 2005 IEEE Int. Jt. Conf. Neural Networks, vol. 3, pp. 1598-1602 (2005)

3. Picard, R.W.: Affective Computing (1997)

4. Fuentes, C., Herskovic, V., Rodríguez, I., Gerea, C., Marques, M., Rossel, P.O.: A systematic literature review about technologies for self-reporting emotional information. J. Ambient Intell. Humaniz. Comput., pp. 1-14 (2016)

5. Zatarain, R., Barrón, M.L., Luis, O.J., Martínez, J.A.: Reconocimiento automático y aspectos éticos de emociones para aplicaciones educativas. Komputer Sapines, México, pp. 27-31 (2014)

6. Cowie, R. et al.: Emotion recognition in human computer interaction. IEEE Signal Process. Mag. 18(1), pp. 32-80 (2001)

7. Steidl, S.: Automatic Classification of Emotion-Related User States in Spontaneous Children's Speech. Universität Erlangen-Nürnberg (2009)

8. González-Hernández, F., Zatarain-Cabada, R., Barrón-Estrada, M.L., Rodríguez-Rangel, H.: Recognition of learning-centered emotions using a convolutional neural network. J. Intell. Fuzzy Syst. (2017)

9. Mehmood, R., Lee, H.; Towards Building a Computer Aided Education System for Special Students Using Wearable Sensor Technologies. Sensors 17(317), pp. 1-22 (2017)

10. Zatarain-Cabada, R., Barrón-Estrada, M.L., González-Hernández, F., Oramas-Bustillos, R., Alor-Hernández, G., Reyes-García, C.A.: Building a Corpus and a Local Binary Pattern Recognizer for Learning-Centered Emotions. Adv. Comput. Intell., vol. II, pp. 524-535 (2017)

11. Zatarain-Cabada, R., Barron-Estrada, M.L., González-Hernández, F., Rodríguez-Rangel, H.: Building a Face Expression Recognizer and a Face Expression Database for an Intelligent Tutoring System. In: Proc. - IEEE 17th Int. Conf. Adv. Learn. Technol. ICALT 2017, no. 2161-377X/17, pp. 391-393 (2017)

12. Zataraín, R., Barrón, M.L., González, F., Reyes-García, C.A.: An Affective and Web 3.0 Based Learning Environment for a Programming Language. Telemat. Informatics (2017)

13. Arana-Llanes, J.Y, González-Serna, G., Pineda-Tapia, R., Olivares-Peregrino, V., Ricarte-Trives, J.J., Latorre-Postigo, J.M.: EEG lecture on recommended activities for the induction of attention and concentration men tal states on e-learning students. J. Intell. Fuzzy Syst. (2017)

14. Bosch N., D’Mello, S.: The Affective Experience of Novice Computer Programmers. Int. J. Artif. Intell. Educ. 27(1), pp. 181-206 (2015)

15. Barrón, M.L., Zatarain, R., Hernández, Y.: Intelligent Tutor with Emotion Recognition and Student Emotion Management for Math Performance. Rev. Electrónica Investig. Educ., vol. 16, pp. 88-102 (2014)

16. Zatarain-Cabada, R., Barron-Estrada, M.L., Ríos-Félix, J.M.: Affective Learning System for Algorithmic Logic Applying Gamification. Chapter Lect. Notes Comput. Sci. Oct. 2017, vol. 576, no. August (2017)

17. Fayyd, U.M., Shapiro, G.P., Padhraic, S.: From data mining to knowledge discovery: An overview. Adv. Knowl. Discov. Data Mining, AAAI Press / MIT Press (1996) 